\title{
A Beat Too Short: A Narrative Review of Heart Failure
}

\author{
Yogesh Acharya $^{1 *}$, Anastasia Anoshina ${ }^{1}$, Daniella Azike ${ }^{1}$, Ranjan Dahal $^{2}$ \\ ${ }^{1}$ Avalon University School of Medicine, Willemstad, Curacao, Netherlands Antilles \\ ${ }^{2}$ Saint Peter's University Hospital, NJ, USA \\ *Corresponding Author: Yogesh Acharya, Avalon University School of Medicine, Willemstad, Curacao, \\ Netherlands Antilles, Tel: +5999 (664)6232; E-mail: dryogeshach@gmail.com
}

Received: 28 October 2018; Accepted: 08 November 2018; Published: 12 November 2018

\begin{abstract}
Background: Heart failure (HF) is one of the most common global public health concerns and a leading cause of death with the steadily increasing prevalence and substantial impact on quality of life. The objective of the article is to analyze various components of HF and discuss its determinants.
\end{abstract}

Materials and Methods: A total of 50 articles was chosen for this literature review from sources such as EBSCO, PUBMED and Google Scholar.

Results: Risk of developing HF is highest with coronary artery disease, diabetes, obesity, hypertension, and smoking respectively. Regardless of the precipitating factors, many compensatory mechanisms in our body serve only as a temporary fix. Most patients present with volume overload and normal or reduced cardiac output. Evaluation begins with a comprehensive history and examination, supported by the laboratory test. The major goals of treatment are to improve quality of life, alleviate symptoms, and reduce morbidity and mortality by reversing or slowing the cardiac and other vital organ dysfunction. Atrial fibrillation and ventricular arrhythmias are the most dreaded complications, and the prognosis is interlinked with associated comorbidities.

Discussion: Currently, prescribed medications can improve the signs and symptoms HF. But it is necessary to develop and validate newer treatments, and early diagnostic modalities to enhance the quality of life. More research is needed to better organize and formulate patient-oriented plans when it comes to non-pharmacological treatment approach. A holistic approach is necessary to curb its growing incidence and address comorbidities, starting with health education and general screening.

Keywords: Heart Failure; Epidemiology; Risk Factors; Prevention; Management; Review 


\section{Introduction}

Globally, heart failure (HF) is considered the leading cause of death and the worldwide prevalence surpasses 37 million [1] with steady growth. Annually, more than 50,000 patients [2] are listed to be candidates for heart transplantation with only as few as 5,000 cardiac allografts available. Undeniably, HF is a worldwide dilemma with a substantial impact on human lives and steadily increasing prevalence [3]. In the US alone, almost 6 million people have HF which will possibly surpass 8 million by $2030[4,5]$. An annual estimated cost of $\$ 30.7$ billion dollars is spent annually, still more than $1 / 3^{\text {rd }}$ die within five years of diagnosis [6].

\section{Materials \& Methods}

The objective of the article is to analyze the different components of HF. A total of 50 articles was chosen for this descriptive review and were retrieved from sources such as EBSCO, PUBMED and Google Scholar. The non patterned search terms included keywords like "Heart Failure", "Epidemiology", "Risk Factors", "Clinical presentation", "Management" and "Recent advancements". The focus was on recent studies that were peer-reviewed and related to HF in humans.

\subsection{Risk factors}

Ischemic heart disease (IHD) is the commonest cause of HF in western countries where as male sex, lack of exercise, cigarette smoking, overweight, diabetes, hypertension, valvular heart disease are all independent risk factors [7]. The risk of developing HF is highest with coronary artery disease (CAD), followed by diabetes, obesity, hypertension and smoking. Similarly, the median time frame (in years) from diagnosis to the development of HF is lowest in CAD followed by diabetes, hypertension and obesity. Females with CAD develop HF earlier than the male counterpart [8].

\subsection{Pathophysiology}

An understanding of the progression of the disease is essential to understand HF. Regardless of the precipitating factors, many compensatory mechanisms in our body serve only as a temporary fix. Decreased cardiac output (CO) represents the initial change, causing inadequate circulation to the peripheral tissues. Blood pressure (BP) subsequently drops activating the sympathetic nervous system to increase the contractility and heart rate (HR). Release of hormones like norepinephrine and the atrial natriuretic peptide is responsible for primary mediators. Increase in contractility is governed by the Frank-Starling's mechanism which states that the force of ventricular contraction is a function of the end diastolic volume (EDV) and muscle length. A subsequent expansion in the length of the sarcomere causes ventricle to dilate. Furthermore, hypertrophy is noted with an increase in the muscular stress leading to apoptosis of the cardiac muscle. This is complicated by activation of the renin-angiotensin-aldosterone system (RAAS), which in turn retain sodium and fluid. This increased reabsorption is meant to correct the venous and arterial pressure and clinically present

as edema. Unfortunately, these compensatory modification increase the blood volume and preload further exacerbating the HF. 


\subsection{Signs and Symptoms}

Most patients present with volume overload and normal CO. Symptoms of volume overload include cough, shortness of breath, leg swelling, increased abdominal girth, orthopnea and paroxysmal nocturnal dyspnea (PND). Healthcare providers use common presenting symptoms for diagnosis; however, atypical presentations at times can be challenging. Many patients may also present with the reduced CO. Symptoms include exertional dyspnea, fatigue, reduced cognition and cold extremities. Typically, patients develop exertional dyspnea followed by orthopnea and PND. Other signs may be evident only at more preceding stages due to compensatory mechanisms. They include tachycardia, weak pulse, pedal edema, raised jugular venous pressure, S3 gallop, crackles, ascites, anasarca and hepatojugular reflux.

\subsection{Diagnosis}

Evaluation begins with a comprehensive medical history and clinical examination, supported by a complete blood count, metabolic profile including serum electrolytes, blood urea nitrogen (BUN), glucose, lipid profile, liver, and thyroid function tests [9]. Other tests include brain natriuretic peptide (BNP) and N-terminal proBNP (NT-proBNP). These neuro-hormones can be utilized as diagnostic and prognostic markers of HF [10,11]. More than $90 \%$ of patients diagnosed with HF present with an abnormal ECG finding [12]. ECG also helps to evaluate ventricular hypertrophy, axis deviation, bundle branch blocks, and atrial enlargements. Arrhythmias are common and include ventricular extra systoles, atrial fibrillations (A-Fib) and ventricular tachycardia (V-Tach). Likewise, chest x-ray (CxR) and echocardiography (ECHO) can be used to evaluate cardiomegaly. Although ECHO provides greater diagnostic utility, $\mathrm{CxR}$ is widely accessible and offer significant cost-benefit [13]. Additionally, measurement of lung function is used to exclude respiratory causes of breathlessness, although the presence of pulmonary disease does not rule out co-existent HF. Epidemiological studies suggest a strong association between obstructive airways and IHD, which is one of the principal causes of HF [14]. Lastly, stress-test and cardiac catheterization can be used for predictive diagnosis, assessing severity and finding underlying etiology.

\subsection{Management}

The major goals of treatment are to improve quality of life, alleviate symptoms and reduce morbidity and mortality by reversing or slowing the cardiac and other major organs dysfunction. Comorbidities contribute to poor prognosis and addressing them with effective management strategies still remains a major challenge. It is important to determine the cause of a targeted therapeutic approach. CAD is the leading cause and after revascularization therapy ventricular function usually improves. Current medical care includes pharmacological \& non-pharmacological approaches, including more invasive practices to limit and if possible reverse the manifestations.

Non pharmacological approaches encompass diet and nutritional control, as well as adequate rest and appropriate exercises. Pharmacological treatment is based on the broad classification into two groups: HF with reduced ejection fraction (HFrEF) or preserved ejection fraction (HFpEF). Acute exacerbations are treated mainly by diuretics, which includes furosemide, bumetanide or torsemide. However, the medical regimen differs significantly based on the etiologies, complications and related comorbidities. Therefore, it is imperative to understand the type of HF before 
starting the precision based treatment.

HFrEF is treated with beta blockers and/or enzyme inhibitors angiotensin converting enzyme inhibitors (ACEI)/angiotensin II receptor blocker (ARB) and/or mineralocorticoid receptor antagonists (MRA) and/or digoxin and/or angiotensin receptor- neprilysin inhibitors (ARNI) and/or Ivabradine. Beta-blockers with mortality benefits are metoprolol succinate [15], carvedilol [16] and bisoprolol [17]. ARB is used if ACEI is not tolerated due to intractable cough. Digoxin is typically used in patients with AF which reduces the risk of hospitalization and improves overall symptoms. MRA is used with NYHA class II-IV after titrating up the doses of ACEI and beta blockers to the maximal level. Based on RALES (Randomized Aldactone Evaluation Study) and Ephesus (Eplerenone post-acute myocardial infarction heart failure and survival study), an addition of the low dose MRA is considered in all patient with moderate to severe chronic HF in the absence of hyperkalemia or significant renal dysfunction or both [18]. Hydralazineisosorbide dinitrate combination is used in patients with chronic kidney disease and those, who are intolerant to ACEI/ARB. In addition, ARNI (Valsartan-sacubitril) can be used in the patient with chronic HFrEF. Pharmacological agents which offer morality benefits are beta- blockers, ACEI or ARB, ANRI, MRA, ivabradine and dinitrate [19]. However, patients with HFpEF are mainly treated with diuretics and antihypertensives. Combination therapy is mainly directed towards improving overall symptoms, and decreasing morbidity and mortality.

When it comes to invasive therapies, an electrophysiologic intervention such as cardiac resynchronization therapydefibrillator (CRT-D) and implantation of cardioverter-defibrillators (ICD) are common. ICD is used for primary prevention of sudden cardiac death in HF. It should only be considered after guideline directed optimized medical therapy for 40 days' post MI and 3 months after revascularization. ICD is recommended for ischemic cardiomyopathy with EF $\leq 35 \%$, and associated HF with NYHA II or III status or EF $\leq 30 \%$ and NYHA I [20]. ICD is recommended for primary prevention of sudden cardiac death in patients with non-ischemic cardiomyopathy with NYHA II-III symptoms, EF $\leq 35 \%$, and failure of guideline-directed medical therapy. Combined CRT-D is recommended for patients with an $\mathrm{EF} \leq 35 \%$, HF with NYHA III or IV status, and a QRS duration $\geq 120 \mathrm{~ms}$ [21, 22].

Heart transplantation is the standard therapy. Mechanical circulatory devices such as ventricular assist devices and total artificial hearts can help bridge the patient till transplantation [23, 24] However, drugs adherence is challenging and poor adherence is mainly contributing to worsening or re-occurrence in most patients [25].

\subsection{Prevention}

There are both pharmacological and non pharmacological preventive modalities. Patients are required to modify the risk factors by changing their lifestyles, diet, weight, stress as well as be physically active, limit alcohol intake and be abstinent from smoking [26]. Based on the comorbid conditions, patients are also placed on specific regimens to improve their health status. For instance, patients who suffer from hypertension and are at high risk for HF can be treated with a thiazide-like diuretic like indapamide, as well as ACEI. Patients unable to tolerate ACEI due to a dry cough, headaches, and hyperkalemia are switched to ARB. These drugs profoundly decrease the patient's potential for 
developing HF, decrease mortality, and can prevent hospitalizations [27]. Beta blockers are other agents with well known beneficial effect. They are useful in patients who experience HFrEF in comparison to those with HFpEF, unstable severe acute HF, and right ventricular failure [28]. Some drugs such as thiazolidinediones and metformin in DM can exacerbate the symptoms of HF, primarily due to fluid retention and lactic acidosis. However, new drugs are being tested, and one of such is a sodium-glucose co-transporter inhibitor (SGLT2). Empagliflozin, a SGLT2, have shown decrement in incidence of cardiovascular events and improvement in patients with type 2 DM and HF [29].

\subsection{Complications}

Arrhythmias, including AF and ventricular arrhythmias, are the most dreaded complications. AF can be seen in about one-fourth of patients that present with chronic HF, but overall ventricular arrhythmias are more common [30]. It can also lead to thromboembolism, which can potentially cause PE, systemic embolization, DVT, stroke, and MI. Gastrointestinal complications like hepatic congestion and hepatic dysfunction are common and muscle weakness or wasting are seen in many patients. HF is frequently associated with impaired kidney function, given their adjoining risk factors of increasing age and associated comorbidities [31]. Similarly, pulmonary edema is seen in more than $3 / 4^{\text {th }}$ of patients with acute HFrEF [32, 33].

\subsection{Prognosis}

The prognostic factors in HF are tightly interlinked with associated comorbidities. In particular, cardiomyopathies with the poor prognosis include low LVEF, secondary MR and HF precipitated by an IHD [34-36]. Studies suggest [37] implementing reverse cardiac remodeling in enhancing cardiac functions leading to a much better outcome. Patients with an increased $\mathrm{EF}$ after receiving this procedure had a better quality of life and 3\% decrease in mortality when compared with the placebo.

\subsection{Recent Advancement}

Sacubitril/Valsartan, previously known as LCZ696, is a neprilysin inhibitor and an ARB, widely used in the treatment of HF at present. Neprilysin normally degrades natriuretic peptide (NP), ergo its inhibition with sacubitril increases the levels of NP and causes vasodilation, the effects of which are counteracted with the valsartan component. In July 2015, Food and Drugs (FDA) approved sacubitril/valsartan for patients with chronic and stable symptomatic HF and who have an $\mathrm{EF}<40 \%$ [38-40]. Furthermore, these agents should be used with other HF treatment modalities but in place of ACEIs or ARBs. Ivabradine is another drug, which decreases HR through the inhibition of funny channels in sinoatrial node. When used in addition to optimal HF medications including beta-blockers it has shown to reduce cardiovascular mortality, hospitalizations and improve quality of life. Based on SHIFT trial [41], it is recommended in patients with HFrEF (EF $\leq 35 \%$ ), HR $\geq 70$ bpm and persisting symptoms, despite optimal medications.

Regarding diagnosis, cardiovascular magnetic resonance (CMR) has become a powerful technique in the preclinical as well as the clinical diagnosis of IHD, CAD, HF, cardiac sarcoidosis, myocardial fibrosis, pericardial sickness, congenital heart problems. It is also helpful to distinguish between acute and chronic MI, is clinically safe, and 
provides an excellent non-invasive diagnostic modality [42-45].

\section{Discussion}

New method of prevention, diagnosis, treatment, and rehabilitation of HF is necessary owing to an increase in the prevalence. Patients with associated comorbidities and pathologies should require specific-to-case therapies, given that certain non-cardiac comorbidities are associated with higher mortality [46, 47]. In addition, physicians are required to correct any treatments based on patient response and potential side effects of certain medications, specifically in elderly, and consider the best options for improvement of life [48].

Furthermore, novel drugs must also be explored to fill the void in treatment modalities. Similarly, more research is required to better organize and create patient-oriented plans when it comes to non-pharmacological treatment approach. The specificity of exercises, proper diet, and meal plans should be established as both preventative and treatment measures [49]. More specific and sensitive tests are needed for early diagnosis before patients succumb to fatal complications.

Newer techniques for imaging and localization of HF, such as the use of Positron Emission Tomography - Cardiac Magnetic Resonance (PET-CMR), has a promising utility but is restricted due to its availability and economic feasibility [50]. It is necessary to develop diagnostic modalities that are economically viable and widely available. Similarly, advancements in stem cell therapy and its utility have many unexplored angles and there are rooms to determine its clinical effectiveness moving forward with the transition from clinical trials into clinical practice [51, 52]. It is utmost important to develop and validate new treatments and diagnostic methods before it's too late. A holistic approach is necessary to curb its growing incidence, starting with health education and general screening.

\section{Conclusion}

Although cardiac pathophysiology, including diagnosis and treatment of HF has become more straightforward, its growing incidence has become a global public health nuisance. Besides the pharmacological treatments, a more holistic approach must be taken into consideration to prevent and treat the patients to decrease the incidence of these varieties of cardiomyopathies. Newer screening and risk assessment modalities should be developed that can be widely applied to the general population. A vigorous initiative is needed to educate people and more resources should be directed towards prevention.

\section{Acknowledgement}

None

\section{References}

1. Ziaeian B, Fonarow GC. Epidemiology and aetiology of heart failure. Nature reviews Cardiology 13 (2016): 
368-378.

2. Taylor DO, Edwards LB, Boucek MM, Trulock EP, Aurora P, Christie J, et al. Registry of the International Society for Heart and Lung Transplantation: twenty-fourth official adult heart transplant report--2007. J Heart Lung Transplant 26 (2007): 769-781.

3. Greenberg B. Gene therapy for heart failure. J Cardiol 66 (2015): 195-200.

4. Mozaffarian D, Benjamin EJ, Go AS, et al. American Heart Association Statistics Committee; Stroke Statistics Subcommittee. Heart Disease and Stroke Statistics-2016 Update: A report from the American Heart Association. Circulation 133 (2016): e38-e360.

5. Savarese G, Lund LH. Global Public Health Burden of Heart Failure. Card Fail Rev 3 (2017): 7-11.

6. Greenberg B. Novel Therapies for Heart Failure. Circ J 80 (2016): 1882-1891.

7. He J, et al. Risk factors for congestive heart failure in US men and women: NHANES I epidemiologic followup study. Arch Intern Med 161 (2001): 996-1002.

8. Dunlay SM, Weston SA, Jacobsen SJ, Roger VL. Risk factors for heart failure: a population-based casecontrol study. Am J Med 122 (2009): 1023-1028.

9. Inamdar AA, Inamdar AC. Heart Failure: Diagnosis, Management and Utilization. Journal of Clinical Medicine 5 (2016): 62.

10. Dassanayaka S, Jones S. Recent Developments in Heart Failure. Circulation Research 117 (2015): e58-e63.

11. Maries L, Manitiu I. Diagnostic and prognostic values of B-type natriuretic peptides (BNP) and N-terminal fragment brain natriuretic peptides (NT-pro-BNP): review article. Cardiovascular Journal of Africa 24 (2013): 286-289.

12. Owusu IK, Boakye YA, Appiah LT. Electrocardiographic Abnormalities in Heart Failure Patients at a Teaching Hospital in Kumasi, Ghana. J Cardiovasc Dis Diagn 2 (2014): 142.

13. Biharas Monfared A, Agha Farajollah S, Sabour F, Farzanegan R, Taghdisi S. Comparison of Radiological Findings of Chest X-Ray With Echocardiography in Determination of the Heart Size. Iranian Red Crescent Medical Journal 17 (2015).

14. Lee H, Liu M, Barrett-Connor E, Wong N. Association of lung function with coronary heart disease and cardiovascular disease outcomes in elderly: The Rancho Bernardo study. Respiratory Medicine 108 (2014): 1779-1785.

15. Hjalmarson A, et al. Effects of controlled-release metoprolol on total mortality, hospitalizations, and wellbeing in patients with heart failure: the Metoprolol CR/XL Randomized Intervention Trial in congestive heart failure (MERIT-HF). MERIT-HF Study Group. JAMA 283 (2000): 1295-1302.

16. Packer $\mathrm{M}$ et al. Effect of carvedilol on the morbidity of patients with severe chronic heart failure: results of the carvedilol prospective randomized cumulative survival (COPERNICUS) study. Circulation 106 (2002): 2194-2199.

17. CIBIS-II Investigators and Committees. The Cardiac Insufficiency Bisoprolol Study II (CIBIS-II): a randomised trial. Lancet 353 (1999): 9-13.

18. Clark D, Guichard J, Calhoun D, Ahmed M. Aldosterone receptor antagonists: current perspectives and 
therapies. Vascular Health and Risk Management 9 (2013): 321-331.

19. Berliner D, Bauersachs J. Current Drug Therapy in Chronic Heart Failure: the New Guidelines of the European Society of Cardiology (ESC). Korean Circ J 47 (2017): 543-554.

20. Al-Khatib SM et al. 2017 AHA/ACC/HRS Guideline for Management of Patients With Ventricular Arrhythmias and the Prevention of Sudden Cardiac Death. Circulation 138 (2018): e272-e391.

21. Stavrakis S, Lazzara R, Thadani $U$. The benefit of cardiac resynchronization therapy and QRS duration: a meta-analysis. J Cardiovasc Electrophysiol 23 (2012): 163-168.

22. Sipahi I, Carrigan TP, Rowland DY, Stambler BS, Fang JC. Impact of QRS duration on clinical event reduction with cardiac resynchronization therapy: meta-analysis of randomized controlled trials. Arch Intern Med 171 (2011): 1454.

23. Ponikowski P et al. 2016 ESC Guidelines for the diagnosis and treatment of acute and chronic heart failure: The Task Force for the diagnosis and treatment of acute and chronic heart failure of the European Society of Cardiology (ESC). Developed with the special contribution of the Heart Failure Association (HFA) of the ESC. Eur J Heart Fail 18 (2016): 891-975.

24. Costanzo M, Ronco C, Abraham W, Agostoni P, Barasch J, Fonarow G et al. Extracorporeal Ultrafiltration for Fluid Overload in Heart Failure. Journal of the American College of Cardiology 69 (2017): 2428-2445.

25. Greenberg B. Novel Therapies for Heart Failure - Where Do They Stand? Circulation Journal 80 (2016): 1882-1891.

26. Djoussé L, Driver J, Gaziano M. Relation between Modifiable Lifestyle Factors and Lifetime Risk of Heart Failure. JAMA 302 (2009): 394.

27. Dupree C. Primary prevention of heart failure: what is the evidence?. Current Opinion in Cardiology 24 (2009): 142-147.

28. Lee H, Baek S. Optimal Use of Beta-Blockers for Congestive Heart Failure. Circulation Journal 80 (2016): 565-571.

29. Swedberg K, Rydén L. Treatment of diabetes and heart failure: Joint Forces. European Heart Journal 37 (2016): 1535-1537.

30. Masarone D, Limongelli G, Rubino M, et al. Management of Arrhythmias in Heart Failure. Schilling JD, ed. Journal of Cardiovascular Development and Disease 4 (2017): 3.

31. Ahmed A, Campbell R. Epidemiology of Chronic Kidney Disease in Heart Failure. Heart Failure Clinics 4 (2008): 387-399.

32. Watson R, Gibbs C, Lip G. ABC of heart failure: Clinical features and complications. BMJ 320 (2000): 236239.

33. Platz E, Jhund P, Campbell R, McMurray J. Assessment and prevalence of pulmonary oedema in contemporary acute heart failure trials: a systematic review. European Journal of Heart Failure 17 (2015): 906-916.

34. Karaye K, Sani M. Factors associated with poor prognosis among patients admitted with heart failure in a Nigerian tertiary medical centre: a cross-sectional study. BMC Cardiovascular Disorders 8 (2008). 
35. Asgar A, Mack M, Stone G. Secondary Mitral Regurgitation in Heart Failure. Journal of the American College of Cardiology 65 (2015): 1231-1248.

36. Shiina Y, Igarashi M, Yoshioka K, Tanabe T, Handa S. Clinical profile and prognosis of hospitalized patients with congestive heart failure in Isehara, Japan. The Tokai Journal of Experimental and Clinical Medicine 30 (2005): 141-148.

37. Reis Filho J, Cardoso J, Cardoso C, Pereira-Barretto A. Reverse Cardiac Remodeling: A Marker of Better Prognosis in Heart Failure. Arquivos Brasileiros de Cardiologia 104 (2015): 502-506.

38. Kaplinsky E. Sacubitril/valsartan in heart failure: latest evidence and place in therapy. Therapeutic Advances in Chronic Disease 7 (2016): 278-290.

39. Struthers A, MacDonald T. Review of aldosterone- and angiotensin II-induced target organ damage and prevention. Cardiovascular Research 61 (2004): 663-670.

40. McMurray J, Packer M, Desai A, Gong J, Lefkowitz M, Rizkala A et al. Angiotensin-Neprilysin Inhibition versus Enalapril in Heart Failure. NEJM 371 (2014): 993-1004.

41. Swedberg K et. al; SHIFT Investigators. Ivabradine and outcomes in chronic heart failure (SHIFT): a randomised placebo-controlled study. Lancet 376 (2010): 875-885.

42. Marcu C, Beek A, van Rossum A. Clinical applications of cardiovascular magnetic resonance imaging. Canadian Medical Association Journal 175 (2006): 911-917.

43. Eitel I, Friedrich M. T2-weighted cardiovascular magnetic resonance in acute cardiac disease. Journal of Cardiovascular Magnetic Resonance 13 (2011): 13.

44. Florian A, Jurcut R, Ginghina C, Bogaert J. Cardiac Magnetic Resonance Imaging in Ischemic Heart Disease A Clinical Review. Journal of Medicine and Life 4 (2011): 330-345.

45. Holzem K. New cardiac magnetic resonance imaging modalities aid in the detection of myocardial fibrosis. Physiological Reports 5 (2017): e13135.

46. Mentz R, Felker G. Noncardiac Comorbidities and Acute Heart Failure Patients. Heart Failure Clinics 9 (2013): 359-367.

47. Widmer F. Comorbidity in Heart Failure. (Herzinsuffizienz und Komorbiditäten). Therapeutische Umschau 68 (2011): 103-106.

48. Zachariah D, Taylor J, Rowell N, Spooner C, Kalra P. Drug therapy for heart failure in older patients—what do they want? Journal of Geriatric Cardiology 12 (2015): 165-173.

49. Al-Mallah M, Sakr S, Al-Qunaibet A. Cardiorespiratory Fitness and Cardiovascular Disease Prevention: an Update. Curr Atheroscler Rep 20 (2018): 1.

50. Quail M, Sinusas A. PET-CMR in heart failure - synergistic or redundant imaging? Heart Failure Reviews 22 (2017): 477-489.

51. Nguyen P, Rhee J, Wu J. Adult stem cell therapy and heart failure, 2000 to 2016: a systematic review. JAMA cardiology 1 (2016): 831-841.

52. Bilgimol J, Ragupathi S, Vengadassalapathy L, Stem cells: An eventual treatment option for heart diseases. World Journal of Stem Cells 7 (2015): 1118-1126. 
Citation: Yogesh Acharya, Anastasia Anoshina, Daniella Azike, Ranjan Dahal. A Beat Too Short: A Narrative Review of Heart Failure. Cardiology and Cardiovascular Medicine 2 (2018): 156-165. 